\title{
Probability distribution of residence times of grains in models of ricepiles
}

\author{
Punyabrata Pradhan and Deepak Dhar \\ Department of Theoretical Physics, Tata Institute of Fundamental Research, Homi Bhabha Road, Mumbai-400005, India
}

\begin{abstract}
We study the probability distribution of residence time of a grain at a site, and its total residence time inside a pile, in different ricepile models. The tails of these distributions are dominated by the grains that get deeply buried in the pile. We show that, for a pile of size $L$, the probabilities that the residence time at a site or the total residence time is greater than $t$, both decay as $1 / t(\ln t)^{x}$ for $L^{\omega} \ll t \ll \exp \left(L^{\gamma}\right)$ where $\gamma$ is an exponent $\geq 1$, and values of $x$ and $\omega$ in the two cases are different. In the Oslo ricepile model we find that the probability that the residence time $T_{i}$ at a site $i$ being greater than or equal to $t$, is a non-monotonic function of $L$ for a fixed $t$ and does not obey simple scaling. For model in $d$ dimensions, we show that the probability of minimum slope configuration in the steady state, for large $L$, varies as $\exp \left(-\kappa L^{d+2}\right)$ where $\kappa$ is a constant, and hence $\gamma=d+2$.
\end{abstract}

\section{INTRODUCTION}

Granular materials have drawn a lot of attention due to their complex flow behaviour under different driving conditions 1]. Slowly driven pile of sand grains serve as a prototype for self-organized criticality (SOC) [2]. Although SOC was not seen in experiments on piles of sand [3], but experiments on piles of long grained rice have shown evidence of power law distribution of avalanche sizes 4, 5, 6]. Historically, perhaps because of its relation to earthquake phenomena, studies of sandpiles [7] have generally focused on the distribution of avalanche sizes. There are only a few theoretical studies of other interesting quantities such as the distribution of total residence times of grains in piles, even though the experimental studies by the Oslo group [4, 5] using coloured tracer grains are now almost a decade old.

In this paper we consider the probability distribution of residence times of grains at a site, and of their total residence times in the pile, in critical slope type slowly-driven sandpile (equivalently ricepile) models. In these models, these residence time distributions are qualitatively different from the critical height type models. In critical height models, the distribution decays exponentially with average total residence time equal to average active mass in the pile 8]. In critical slope models, there is a possibility that the grain gets buried very deep in the pile, and then takes a long time to come out. We shall show that this makes the cumulative probability distribution of these residence times to have a characteristic $1 / t$ decay for large times $t$, (modified by a logarithmic multiplicative correction factor), independent of details of the toppling rules, and of the dimensionality of the system.

There have been some numerical and analytical studies of these distributions earlier. Frette has proposed a theoretical model, called the Oslo ricepile model [9], which seems to reproduce the phenomenology of the ricepile experiment well. In the experimental studies of ricepiles, Christensen et. al. [4, 5] estimated that average total residence time of grains in a pile of size $L$ varies as $L^{\nu}$, where they estimated $\nu=1.5 \pm 0.2$. From numerical simulations of the Oslo model for systems of size $L \leq 1600$, the exponent characterizing the power law decay of the probability density of total residence times at large times was estimated as 2.2 \pm 0.1 . Boguna and Corral [10], and Carreras et. al. [11] have used a continuous-time random walk model of the motion of grains, with long trapping times and a power-law distribution of step sizes, to explain the anomalous diffusion of tracer grains. In earlier papers 8, 12], we have studied the total residence time distribution in the critical height type sandpile models with both deterministic and stochastic toppling rules. We reduced the problem to a diffusion problem of a single particle in a medium with space dependent jump rates and showed that the distribution of the total residence time does not have any power law tail. We also obtained the non-universal scaling form of the distribution which depends on the probability distribution of where grains are added into the pile.

In this paper we study the distribution of total residence times of grains in the pile, and also of residence time at a site, in a class of critical slope type sandpile models. We define the residence time $T_{i}$ at a site $i$ is the time spent by a grain at the this site, measured in units of the time interval between successive addition of grains. The total residence time $T$ is defined similarly. We show that the probability of the residence time at a site or the total residence time in the pile, being greater than or equal to $t$, decays as $1 / t(\ln t)^{\delta}$ for a very wide range of $t$. The upper cutoff in both the distributions scales with system size $L$ as $\exp \left(\kappa L^{\gamma}\right)$ where $\gamma$ is an exponent $\geq 1$ and $\kappa$ is a positive constant.

For the Oslo ricepile model, we find an unexpected behaviour in the cumulative probability that a grain staying at a site $i$ at least upto time $t$, is not a monotonically increasing function of system size $L$. We argue that this implies the cumulative probability distribution function $\operatorname{Prob}_{L}\left(T_{1} \geq t\right)$ cannot have a simple finite size scaling form. We show that $\gamma=d+2$ in $d$ dimensions for this model.

Plan of the paper is as follows. In section 2 we define 
the four models studied in this paper. In section 3 we present the simulation results for the residence time $T_{1}$ at site 1 for the $1 d$ Oslo ricepile model and explain the non-monotonic behaviour of the cumulative distribution $\operatorname{Prob}_{L}\left(T_{1} \geq t\right)$ with $L$ by relating the residence times of grains at the site 1 to the statistical properties of height fluctuation at that site. We also explain the origin of multiplicative logarithmic correction factor appearing in the $1 / t$ decay of $\operatorname{Prob}_{L}\left(T_{1} \geq t\right)$. In section 4 we discuss the $1 / t$ power law form of $\operatorname{Prob}_{L}(T \geq t)$, where $T$ is the total residence times, for large $t$ in the $1 d$ Oslo model and show that this also has a multiplicative logarithmic correction. In section 5 we argue that the probability of minimum slope configuration occurring in the steady state of the $1 d$ Oslo ricepile model, scales with system size $L$ as $\exp \left(-\kappa L^{3}\right)$ where $\kappa$ is some positive constant. In section 6 we present our simulation results for other models and show that in all cases the cumulative distributions is qualitatively similar to the $1 d$ Oslo ricepile model. The last section contains a summary and some concluding remarks.

\section{DEFINITION OF THE MODELS}

We consider general critical slope type sandpile models where the configurations are specified by integer height variables $h(\vec{x})$, i.e., number of grains, at any site $\vec{x}$ of a finite $d$-dimensional lattice. Whenever height difference between two adjacent sites is greater than a threshold value, some specified number of grains are transferred to the neighbouring sites. Piles are driven by adding grains, one at a time, at a fixed, or at a randomly chosen site. Grains are added only when there are no unstable sites left in the system, and can leave the pile from the boundary. We update all unstable sites in parallel. We have studied four different models both in one and two dimensions : the Oslo ricepile model and it's $2 d$ generalization, local limited model and it's variation. We now define the precise rules of these four models.

\section{Model-A: The Oslo ricepile model.}

The Oslo ricepile model $[\underline{5}$ is defined as follows. We consider a one dimensional ricepile, which is specified by an integer height variable $h_{i}$ at each site $i$ of a onedimensional lattice, with $1 \leq i \leq L$. The slope at site $i$ is defined to be $h_{i}-h_{i+1}$. Whenever the slope $z_{i}$ at any site $i$ is higher than a critical value $z_{c, i}$, the site becomes unstable and one grain from the unstable site goes to the right neighbour, i.e., $h_{i} \rightarrow h_{i}-1$ and $h_{i+1} \rightarrow h_{i+1}+1$. Whenever there is a toppling at site $i, z_{c, i}$ is randomly, independent of the history, reset to one of the two values, 1 and 2, with probability $q$ and $p$ respectively, where $p+q=1$. Whenever there is a toppling at site $i=L$ (rightmost end), one grain goes out of the system. Grains are added only at site 1 .

The $1 d$ Oslo ricepile model has an abelian property 13. The final height configuration does not depend on the order we topple the unstable sites. After addition of total $L(L+1)$ grains, the pile reaches the critical steady state [13]. Since we have chosen the values of $z_{c}$ to be 1 or 2 , in the steady state height profile fluctuate between slope 1 and 2. For number of sites $L$, the number of possible configurations in the critical states are exponentially large, approximately $\frac{1+\sqrt{5}}{2 \sqrt{5}}\left(\frac{3+\sqrt{5}}{2}\right)^{L}$, for large $L$ 14. The probabilities of various configurations in the steady state differ from one another by many orders of magnitude unlike the BTW model

\section{Model-B : $2 d$ generalization of the Oslo model.}

The Oslo model defined above can easily be generalized to two dimensions. We take a triangular region of a square lattice, the sites of which are indexed by $(i, j)$ with $i, j \geq 1$ and $i+j \leq L+1$. The height of the pile at site $(i, j)$ is denoted by $h(i, j)$. Whenever the height difference between site $(i, j)$ and any of it's neighbouring sites exceeds a critical value $z_{c}(i, j)$, assigned to the site $(i, j)$, there is a toppling at site $(i, j)$ and one grain is transferred from this site to the lower neighbouring site towards the unstable direction. If there are more than one unstable directions, grain is transferred towards the greatest slope. If the two directions have equal slope values, one grain is transferred randomly towards any one of these two directions. Whenever there is a toppling at a site $(i, j), z_{c}(i, j)$ is reset randomly, independent of the history, to either 2 or 1 with probability $p$ or $q$ respectively, where $p+q=1$. One grain is lost, whenever there is a toppling at the boundary sites i.e., along $i+j=L+1$ line. The model defined above in two dimensions is not abelian because final stable configuration depends on the order we topple the unstable sites. Grains are added only at the corner site $(1,1)$.

\section{Model-C : The local limited model.}

The local limited model [15] is a one dimensional model defined as follows. The slope $z_{i}$ is defined as i.e., $z_{i}=$ $h_{i}-h_{i+1}$. Whenever value of the slope $z_{i}$ at any site $i$ is higher than a critical value $z_{c}$, which we choose to be 2, the site becomes unstable and two grains from the unstable site goes to the right neighbour, i.e., $h_{i} \rightarrow h_{i}-2$ and $h_{i+1} \rightarrow h_{i+1}+2$. Slope at any site may be negative in the local limited model. Whenever there is a toppling at site $i=L$ (rightmost end), two grains go out of the system simultaneously.

Grains are added uniformly everywhere. This model is also not abelian. It is easy to see that in this case 


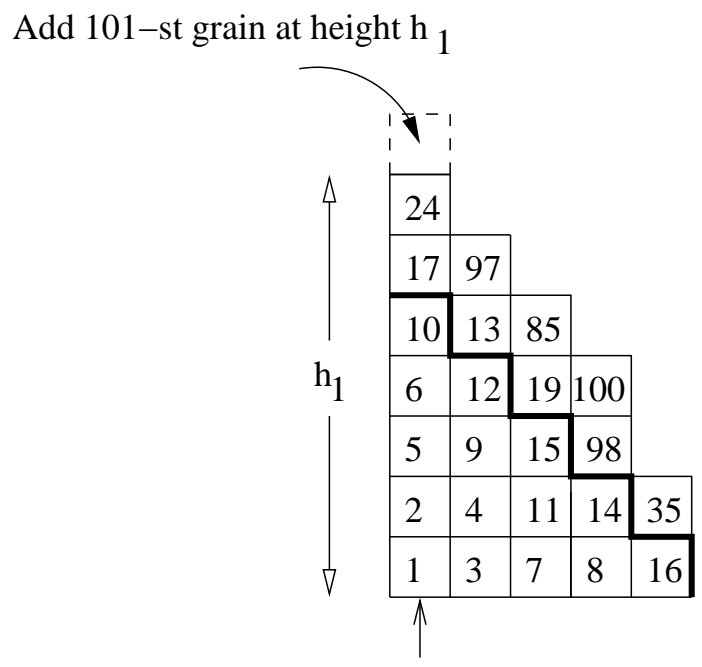

Stack at site $\mathrm{i}=1$

FIG. 1: Rice pile of size $L=5$ after addition of 100 grains. All grains are numbered whenever added in the pile. Minimum slope is denoted by the thick line.

the maximum and the minimum slopes are 2 and 1 respectively. Total number of recurrent configurations in the steady state can be determined exactly, and varies as $\frac{4^{L}}{L^{3 / 2}}$ for large $L[16]$. It is known that the probabilities of occurrence of various configurations in the steady state is not equal, and may differ from one another by many orders of magnitude.

\section{Model-D : Model with non-nearest neighbour transfer of grains.}

Model-D is a variation of the model-C [15]. Whenever value of the slope $z_{i}>2$ at any site $i$, the site becomes unstable and two grains from the unstable site are transferred to the right, one grain transferred to site $i+1$ and the other one transferred to site $i+2$, i.e., $h_{i} \rightarrow h_{i}-2, h_{i+1} \rightarrow h_{i+1}+1$ and $h_{i+2} \rightarrow h_{i+2}+1$. If there is a toppling near the right boundary, grain goes out of the pile. The order we relax unstable sites matters. The grains are added uniformly everywhere. The local slope can be negative as in model-C. The minimum and maximum slope in this model are also 1 and 2 respectively.

The pile in all four cases is driven slowly, by adding one grain per unit time, starting with the initial configuration of height zero at all sites. We assume that the time interval between addition of two grains is chosen long enough so that all avalanche activity has died before a new grain is added. The grain added at time $n$ will be labeled by the number $n$. We think of the grains at a particular site as stacked vertically, one above the other (Fig. 1). Whenever a grains is added at a site, it sits on the top of the stack. When one unstable grain leaves the stack, it is taken from the top of the stack. In model-C, when two grains leave a site, we first take out the topmost grain from the site and put it on the top of right nearest neighbour stack, then we take the second unstable grain and put it on the top of the first grain at right nearest stack. In model-D, we transfer the unstable grain, second from the top, to the right nearest neighbour and transfer the topmost one to next to the right nearest neighbour.

If a particular grain $n$ enters a site $i$ at time $t_{i n}(i, n)$ and leaves the site at time $t_{\text {out }}(i, n)$, it's residence time $T_{i}(n)$ at site $i$ is defined as the time spent by the grain at the site $i$, i.e., $T_{i}(n)=t_{\text {out }}(i, n)-t_{\text {in }}(i, n)$. The residence time of the $n^{\text {th }}$ grain, $T(n)$, is the total time spent by the grain inside the pile. For a directed ricepiles in one dimension where grains move only in one direction and by one step in each toppling, the residence time $T(n)$ equals to $\sum_{i=1}^{L} T_{i}(n)$ (e.g. in model-A and model-C). We define the function $\operatorname{Prob}_{L}\left(T_{j} \geq t\right)$ as the probability that a new grain added in the steady state of the pile will have a residence time at site $j$ is greater than or equal to $t$, and $\operatorname{Prob}_{L}(T \geq t)$ as the probability that its total residence time in the pile is greater than or equal to $t$. Clearly, we have $\operatorname{Prob}_{L}\left(T_{j} \geq 0\right)=\operatorname{Prob}_{L}(T \geq 0)=1$.

\section{RESIDENCE TIMES AT THE FIRST SITE IN THE OSLO RICEPILE MODEL}

The qualitative behaviour of distributions $\operatorname{Prob}_{L}\left(T_{i} \geq\right.$ $t$ ) for $i=1$ can be seen in the simulation results shown in Fig. 2 and Fig. 3. We have done our simulations for $p=q=\frac{1}{2}$ and different system sizes, $L=20,25,35$ and 50 . We averaged the data for a total $10^{9}$ grains added in the pile for each $L$. Fig. 2 shows the plot of $\operatorname{Prob}_{L}\left(T_{1} \geq\right.$ $t$ ) versus time $t$ for different values of $L$. Interestingly, various curves for different $L$ have steps like structures. The curves for different values of $L$ cross each other many times. The unusual non-monotonic behaviour is not an artifact of statistical fluctuations. The statistical errors in the data are much smaller than the step sizes except in the tail region (i.e, $t \gg 10^{6}$ ). The crossing of the curves for the cumulative probabilities persists for quite large system sizes also. In Fig. 3, we have plotted $\operatorname{Prob}_{L}\left(T_{1} \geq\right.$ $t$ ) versus $t$ for two much bigger system sizes, $L=100$ and $L=200$. We see that in this case also the probability that a grain remains in the pile of size $L=100$, for time greater than or equal to $6 \times 10^{5}$, is higher by a factor 1.8 than for a pile, two times larger size $L=200$. Steps like structures are not $\log$ periodic as the height and width of a step in each curve increases when going down the curve even on the log scale. The existence of several steps, whose positions and logarithmic widths are different for different $L$ 's, implies that simple finite size 


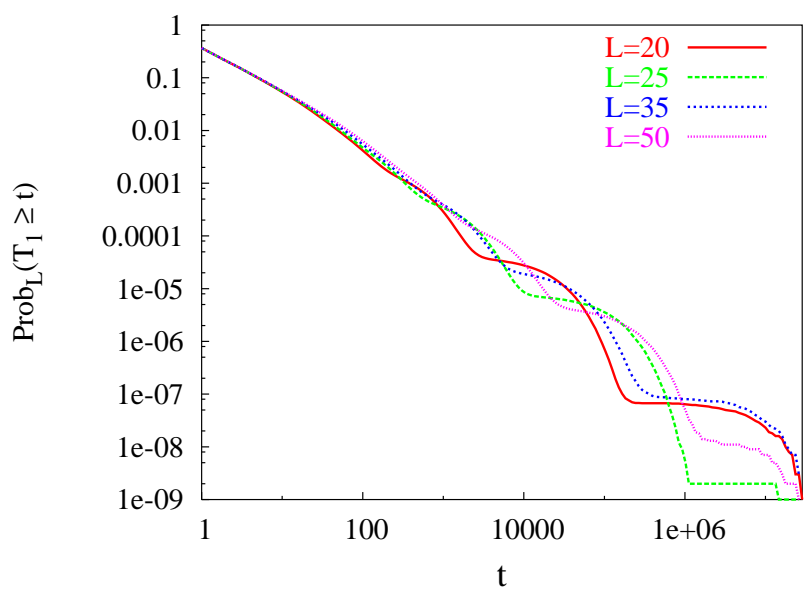

FIG. 2: The cumulative probability $\operatorname{Prob}_{L}\left(T_{1} \geq t\right)$ versus time $t$ for lattice sizes $L=20,25,35$ and 50 in the $1 d$ Oslo ricepile model. Total $10^{9}$ grains were added.

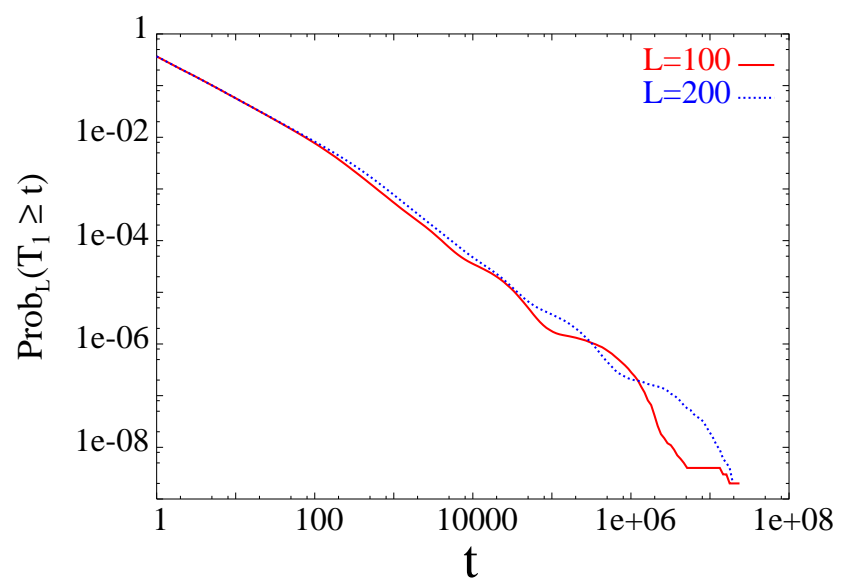

FIG. 3: The cumulative probability $\operatorname{Prob}_{L}\left(T_{1} \geq t\right)$ versus time $t$ for lattice sizes $L=100$ and $L=200$ in the $1 d$ Oslo ricepile model.

scaling cannot hold in this case.

\section{Relationship between residence times $T_{1}$ and height fluctuations.}

We can understand the residence time distribution of grains at any site in terms of the fluctuation of height at that site. The $h_{i}(t)$ be the height of the pile at a site $i$ just after the $t^{t h}$ grain has been added. This is a stochastic process and, in the steady state, it fluctuates in time between a upper bound, $h_{\max }$, and a lower bound, $h_{\min }$. In case of the height fluctuation at site $1, h_{\max }=2 L$ and $h_{\min }=L$. The height $h_{1}(t)$ at the site 1 has a stationary probability distribution which is sharply peaked near its average value $\bar{h}_{1}$, and has the width $\sigma_{h_{1}}$ which is standard deviation of the fluctuation of height $h_{1}$. In the steady

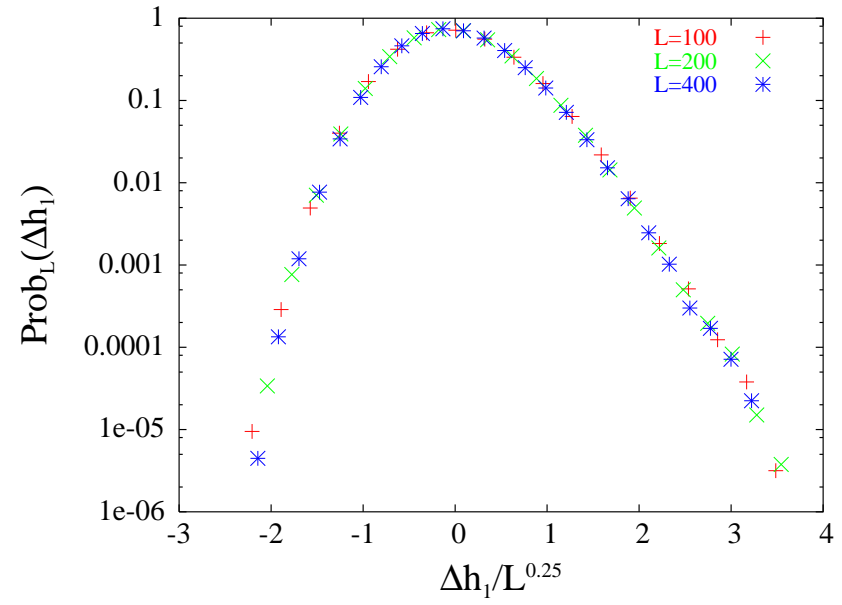

FIG. 4: Scaling collapse of various probability distributions $\operatorname{Prob}_{L}\left(\Delta h_{1}\right)$ where $\Delta h_{1}$ is the deviation of height at site 1 about it's average value, for different system sizes, $L=$ 100, 200 and 400 for the $1 d$ Oslo ricepile model.

state, the average value of $h_{1}$ varies as $L$, and the width $\sigma_{h_{1}}$ varies as $L^{\omega_{1}}$, where exponent $\omega_{1}<1$. For large $L$, the probability distribution of $h_{1}$ has a scaling form as given below.

$$
\operatorname{Prob}_{L}\left(h_{1}\right)=L^{-\omega_{1}} g\left(\frac{h_{1}-\bar{h}}{L^{\omega_{1}}}\right)
$$

In Fig. 4 we have shown a scaling collapse of various probability distribution of height at site $1, \operatorname{Prob}_{L}\left(\Delta h_{1}\right)$, where $\Delta h_{1}=h_{1}-\bar{h}_{1}$, for various values of system sizes, $L=100,200$ and 400 in the $1 d$ Oslo ricepile model. We get a good collapse using the scaled variable $\Delta h / L^{\omega_{1}}$ where $\omega_{1} \approx 0.25$. Here the scaling function $g(x)$ is nearly Gaussian for $x$ near zero. But very large deviations of $h_{1}$ from the mean value are not well-described in the Gaussian approximation. Later we shall argue that in the Oslo model scaling function $g(x)$ varies as $\exp \left(-|x|^{\frac{1}{1-\omega_{1}}}\right)$ for $x \gg 1$ and it varies as $\exp \left(-|x|^{\frac{3}{1-\omega_{1}}}\right)$ for $x \ll-1$.

Let us consider variation of height $h_{1}$ at the first site with time $t$ shown schematically in Fig. 5. Note that $h_{1}(t)$ is piecewise constant line segments, with possible jumps at the integer time $t$. The value of $h_{1}(t)$ at time $t$ is denoted by $y$-coordinate of the line segment which is just at the right of the coordinate $t, e . g ., h_{1}(0)=33$, $h_{1}(1)=34$, etc. A grain added at time $t$, when the height at the first site is $h_{1}(t-1)$, leaves the site at time $t^{\prime}$, we must have $h_{1}\left(t^{\prime}\right) \leq h_{1}(t)$, and $h_{1}\left(t^{\prime \prime}\right)>h_{1}(t)$, for all $t^{\prime \prime}$ satisfying $t<t^{\prime \prime}<t^{\prime}$. As an example, for the time series of $h_{1}(t)$ shown in Fig. 5 , the grain added at $t=14$ stays at site 1 upto time $t=41$ and then goes out of the site 1 at $t=42$ (i.e., just after addition of the $42 n d$ grain), and so $T_{1}(14)=28$. As $h_{1}(13)=h_{1}(12)$, the grain added at $t=12$ comes out immediately, and hence $T_{1}(12)=0$. 


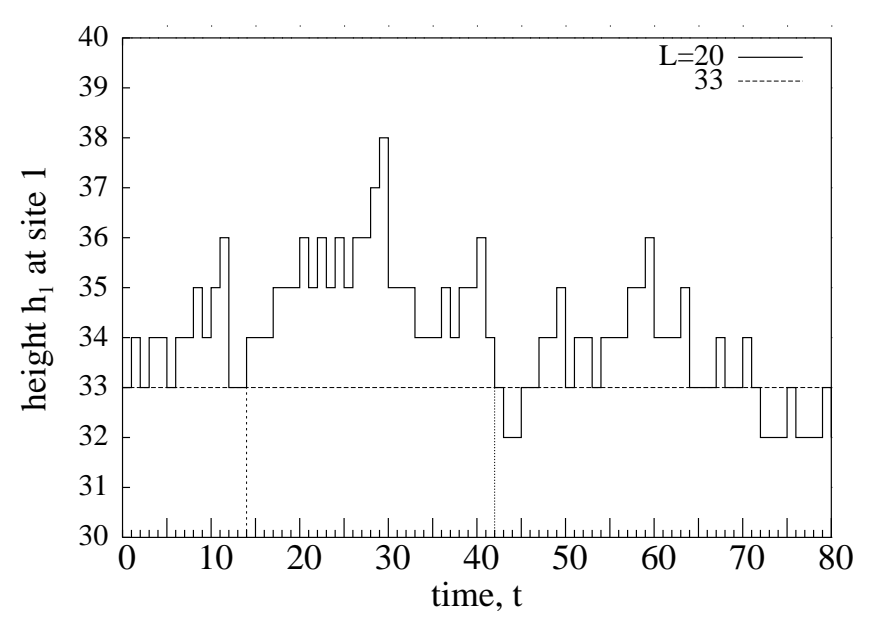

FIG. 5: Time fluctuation of height at first site is plotted as a function of time in the $1 d$ Oslo ricepile model for $L=20$. The horizontal line is at $h_{1}=33$ which is the maximum probable height. The first and second vertical lines are at $t=14$ and $t=42$ respectively.

Let $\operatorname{Prob}_{L}\left(T_{1} \geq t \mid h_{1}\right)$ be the conditional probability that a grain stays at site 1 for time greater than $t$, given that it was added when the height was $h_{1}$. Since $\operatorname{Prob}_{L}\left(h_{1}\right)$ is the probability that height was $h_{1}$ when the grain was added, we have the following, summing over all possible values of $h_{1}$.

$$
\operatorname{Prob}_{L}\left(T_{1} \geq t\right)=\sum_{h_{1}=h_{m i n}}^{h_{\max }} \operatorname{Prob}_{L}\left(h_{1}\right) \operatorname{Prob}_{L}\left(T_{1} \geq t \mid h_{1}\right)
$$

But $\operatorname{Prob}_{L}\left(T_{1}>t \mid h_{1}\right)$ can also be written as the conditional probability that the height of the pile at site 1 would remain above $h_{1}$ for an interval $\geq t$, given that the height is $h_{1}$ in the steady state. This probability can be calculated from the general theory of Markov chains as the probability of first return to a height less than or equal to $h_{1}$, given that we start with height $h_{1}$ in the steady state, and add one grain per unit time. The probability that no return has occurred up to time $t$ decreases as $\exp \left[-\lambda\left(h_{1}\right) t\right]$ for large $t$, where $\lambda\left(h_{1}\right)$ is the largest eigenvalue of the the reduced Markov matrix, with rows and columns corresponding to configurations with heights at site 1 , below or equal to $h_{1}$, removed [17, 18]. While it is not very easy to calculate $\lambda\left(h_{1}\right)$ exactly, clearly it decreases as $h_{1}$ decreases. For $h_{1}=h_{\max }$, it is $+\infty$ as the height at the site cannot be higher than $h_{\max }$ and $T_{1}$ must always be zero. Also it is very small for $h_{1}$ near $h_{\text {min }}$, as the pile returns to very low values of $h_{1}$ only rarely.

For large $t$, in the sum in r.h.s. of Eq.(2), only terms with $h_{1}$ near $h_{\min }$ make a significant contribution. In this case, it is a reasonable approximation to replace the function $\operatorname{Prob}_{L}\left(T_{1}>t \mid h_{1}\right)$ by a simple exponential, with $\lambda\left(h_{1}\right)=\left\langle T_{1}\right\rangle_{h_{1}}$. Thus we write, for large $t$,

$$
\operatorname{Prob}_{L}\left(T_{1}>t \mid h_{1}\right) \simeq \exp \left(-t /\left\langle T_{1}\right\rangle_{h_{1}}\right)
$$

It is easy to write the conditional expectation value of the residence time at the first site, $\left\langle T_{1}\right\rangle_{h_{1}}$, given that the grain was added at the height $h_{1}$ in terms of the stationary probability distribution $\operatorname{Prob}_{L}\left(h_{1}\right)$ exactly as

$$
\left\langle T_{1}\right\rangle_{h_{1}}=\frac{\operatorname{Prob}_{L}\left(\text { height }>h_{1}\right)}{p_{1} \operatorname{Prob}_{L}\left(h_{1}\right)}
$$

where $p_{1}$ is the probability of adding a grain at site $i=1$. When we add grains only at first site, $p_{1}=1$ and when we add grains uniformly everywhere, $p_{1}=1 / L$.

Proof : Define an indicator function $\eta_{n, t}=1$, if the $n^{\text {th }}$ grain is at height $h_{1}$ at time $t$, and zero otherwise. Clearly, the sum of $\eta_{n, t}$ over $t$ is the residence time of $n^{\text {th }}$ grain at height $h_{1}$. Then, averaging over $n$ we get the mean residence time. But the sum of $\eta_{n, t}$ over $n$ and $t$ both gives a contribution whenever there is a grain at height $h_{1}$, and hence is equal to $\operatorname{NProb}_{L}\left(\right.$ height $\left.\geq h_{1}\right)$ where $N$ is total number of grains added and $N$ is very large. Dividing this sum by average number of grains added at height $h_{1}$, which is equal to $p_{1} N \operatorname{Prob}_{L}\left(h_{1}\right)$, we get $\left\langle T_{1}\right\rangle_{h_{1}}$. Hence, Eq.(4) follows.

We substitute this estimate of $\left\langle T_{1}\right\rangle_{h_{1}}$ in Eq. (3). We note that for large $t$, the terms in the summation that contribute significantly correspond to $h_{1}$ near $h_{\min }$. For these values of $h_{1}$, Prob(height $>h_{1}$ ) is nearly 1 , and $\left\langle T_{1}\right\rangle_{h_{1}}$ may be replaced, with small error, by $1 / \operatorname{Prob}_{L}\left(h_{1}\right)$ (see Eq. 4). Then Eq. (2) can be approximately written as given below.

$$
\operatorname{Prob}_{L}\left(T_{1} \geq t\right) \simeq \sum_{h_{1}=h_{m i n}}^{h_{\max }} \operatorname{Prob}_{L}\left(h_{1}\right) e^{-t \operatorname{Prob}_{L}\left(h_{1}\right)}
$$

Thus, the distribution of residence times $T_{1}$ can be expressed in terms of the probability distribution $\operatorname{Prob}_{L}\left(h_{1}\right)$ of height $h_{1}$.

\section{Behaviour of $\operatorname{Prob}_{L}\left(T_{1} \geq t\right)$ for large $t$.}

Now we shall use the knowledge of the behaviour of $\operatorname{Prob}_{L}\left(h_{1}\right)$ to explain the step-like structures in the distribution function $\operatorname{Prob}_{L}\left(T_{1} \geq t\right)$.

For $h_{1} \ll \bar{h}_{1}$, the probability distribution of height $\operatorname{Prob}_{L}\left(h_{1}\right)$ falls very rapidly. Actually, it will be argued in section 5 that for $h_{1} \ll \bar{h}_{1}$ the ratio $\operatorname{Prob}_{L}\left(h_{1}-\right.$ $1) / \operatorname{Prob}_{L}\left(h_{1}\right)$ is of order $\exp \left(-a L^{2}\right)$ where $a$ is a constant and hence is very much less than 1 . The values of $\operatorname{Prob}_{L}\left(h_{1}\right)$ for different $h_{1}$ 's could differ by several orders of magnitude from each other, if $h_{1}$ is sufficiently 


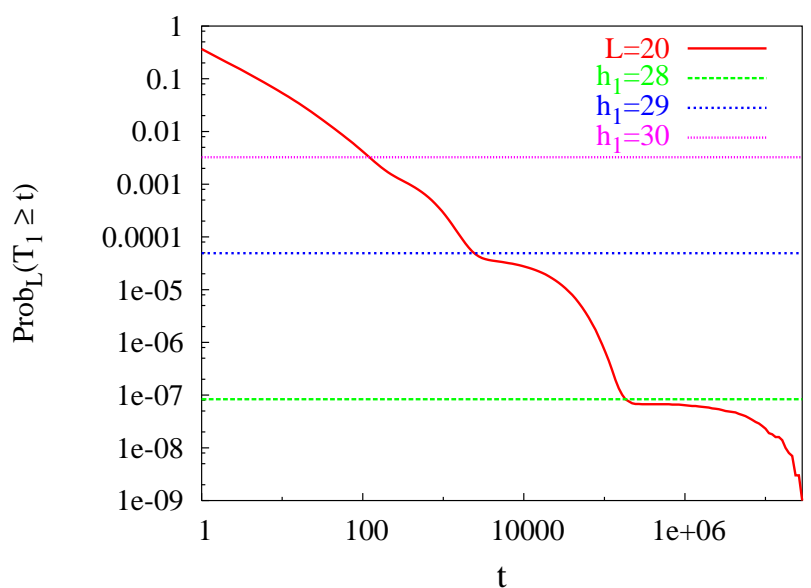

FIG. 6: The cumulative probability $\operatorname{Prob}_{L}\left(T_{1} \geq t\right)$ versus time $t$ for lattice size $L=20$.

near $h_{\text {min }}$. Now in the interval of $1 / \operatorname{Prob}_{L}\left(h_{1}-1\right) \gg$ $t \gg 1 / \operatorname{Prob}_{L}\left(h_{1}\right)$, only a single term corresponding to $h_{1}$ contributes significantly to the summation, and then the summation is nearly independent of $t$. It is clearly seen from Fig. 6, where $\operatorname{Prob}_{L}\left(T_{1} \geq t\right)$ is plotted $t$ for $L=20$ in the $1 d$ Oslo model. We can identify three steps in the plot. Each step in the curve can be associated with a unique value of $h_{1}\left(h_{1}=28,29\right.$ and 30) and steps appear at the corresponding value of $\operatorname{Prob}_{L}\left(h_{1}\right)$ along the $y$-axis.

This explains the steps like structure of $\operatorname{Prob}_{L}\left(T_{1} \geq t\right)$ as a function of $t$. Also, the function decays roughly as $1 / t$ since we must have $\operatorname{Prob}_{L}\left(h_{1}\right) \sim 1 / t$ for the term to contribute in Eq. (5). If $h_{1}^{*}(t)$ is the value of $h_{1}$ that contributes most in Eq. (5), the value of $h_{1}^{*}(t)$ is given by the condition $\operatorname{Prob}_{L}\left(h_{1}^{*}(t)\right) \approx 1 / t$. Substituting this condition in Eq. (11), we get

$$
g\left(\frac{h_{1}^{*}(t)-\bar{h}}{L^{\omega_{1}}}\right) \approx L^{\omega_{1}} / t
$$

Thus the $t$ dependence of $h_{1}^{*}(t)$ comes through the scaling variable $t L^{-\omega_{1}}=\tau$ (say). Then for $\tau$ large the argument establishing the $1 / t$ dependence of $\operatorname{Prob}_{L}\left(t_{1}>t\right)$ given above is quite robust. However more careful analysis of Eq. (57) shows that there is also a logarithmic multiplicative correction factor with the $1 / t$ decay of $\operatorname{Prob}_{L}\left(T_{1} \geq t\right)$.

For large $L$ and $t$, the terms, which contribute to $\operatorname{Prob}_{L}\left(T_{1} \geq t\right)$ in Eq. (5), correspond to the values of $h_{1}$ for which $h_{1} \ll \bar{h}_{1}$. Substituting the scaling form of $\operatorname{Prob}_{L}(h)=\frac{1}{L^{\omega_{1}}} g\left(\frac{h-\bar{h}_{1}}{L^{\omega_{1}}}\right)$ (see Eq. (10) in Eq (15) and putting $x=\left(h-\bar{h}_{1}\right) / L^{\omega_{1}}$, we get the following.

$$
\operatorname{Prob}_{L}\left(T_{1} \geq \tau L^{\omega_{1}}\right) \sim \int d x g(x) \exp [-g(x) \tau]
$$

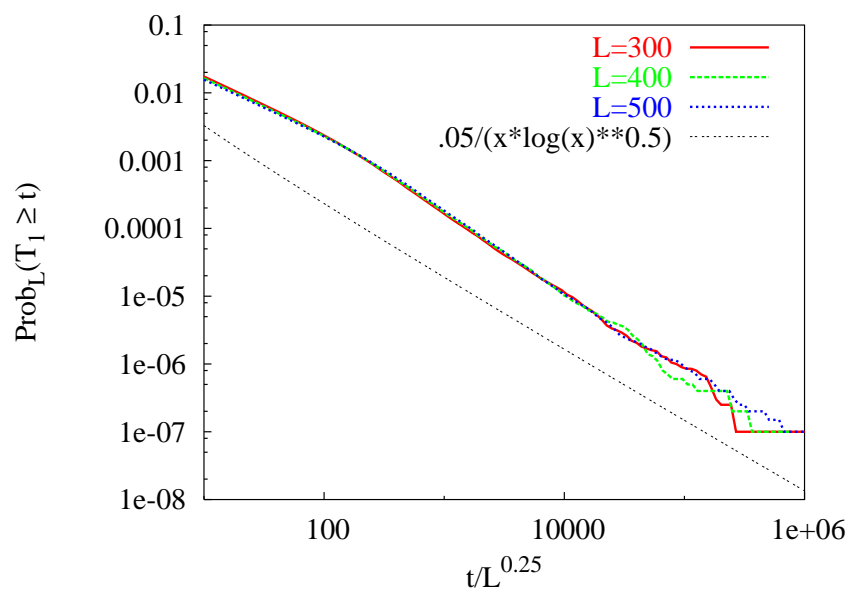

FIG. 7: The cumulative probability $\operatorname{Prob}_{L}\left(T_{1} \geq t\right)$ for residence time at the first site has been plotted against the scaled residence time $t / L^{0.25}$ for lattice sizes $L=300,400$ and 500 in the $1 d$ Oslo ricepile model. Total $10^{7}$ grains were added.

where $\tau=t / L^{\omega_{1}}$. We have assumed that the probability distribution $\operatorname{Prob}_{L}\left(h_{1}\right) \ll 1$, but is not rapidly decaying so that $\operatorname{Prob}_{L}\left(h_{1}-1\right) / \operatorname{Prob}_{L}\left(h_{1}\right) \approx 1$, and then the summation of Eq. (5) can be replaced by an integral over the scaled variable $x$. Actually in the real simulation (or experiment) for large $L$, this is the region of $t$ we explore, we cannot go too far down the tail of $\operatorname{Prob}_{L}\left(T_{1} \geq t\right)$.

Now it is easy to see the origin of logarithmic correction if we choose a particular form of the scaling function $g(x)$ as $\exp \left(-|x|^{\alpha}\right)$ for $x \ll 1$, with $\alpha>0$, and try to find out the large $t$ behaviour of the above equation in terms of the scaling variable $\tau=t / L^{\omega_{1}}$. We first substitute $s=\exp \left(-|x|^{\alpha}\right)$ in Eq (7) and get,

$$
\operatorname{Prob}_{L}\left(T_{1} \geq \tau L^{\omega_{1}}\right) \sim \frac{1}{\alpha} \int \frac{d s}{[-\ln (s)]^{\frac{\alpha-1}{\alpha}}} \exp (-s \tau)
$$

The asymptotic behaviour of the of the above integral for $\tau$ large is easy to evaluate, giving

$$
\operatorname{Prob}_{L}\left(T_{1} \geq \tau L^{\omega_{1}}\right) \sim 1 / \tau(\ln \tau)^{\frac{\alpha-1}{\alpha}}
$$

In Fig. 7 we have plotted $\operatorname{Prob}_{L}\left(T_{1} \geq t\right)$ against scaled variable $t / L^{\omega_{1}}$ with $\omega_{1}=0.25$ for large values of system sizes $L=300,400$ and 500 in the $1 d$ Oslo model. We fit the scaled curves with a functional form given in Eq. (9) with $\alpha=2$ since the scaling function $g(x)$ is Gaussian near $x=0$.

Now it is clear that there is a logarithmic multiplicative factor in $1 / t$ decay and we take account of this logarithmic multiplicative correction by writing the cumulative probability as given below.

$$
\operatorname{Prob}_{L}\left(T_{1} \geq t\right) \simeq L^{\omega_{1}}\left[t \log ^{\delta_{1}}\left(t L^{-\omega_{1}}\right)\right]^{-1}
$$




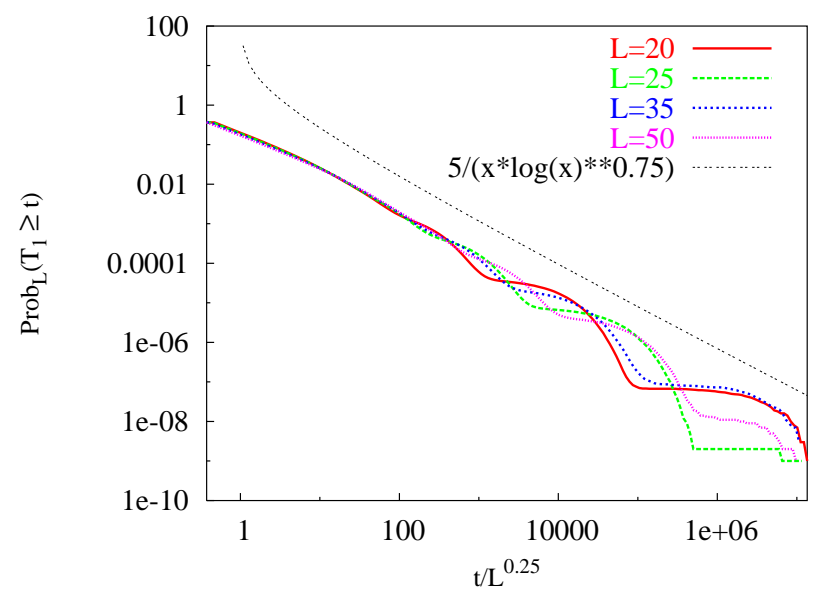

FIG. 8: The cumulative probability $\operatorname{Prob}_{L}\left(T_{1} \geq t\right)$ for residence time at the first site has been plotted against the scaled time $t / L^{0.25}$ for lattice sizes $L=20,25,35$ and 50 in the $1 d$ Oslo ricepile model. Total $10^{9}$ grains were added.

where we have used the fact that the answer is function of the scaling combination $t L^{-\omega_{1}}$ and $\delta_{1}=(\alpha-1) / \alpha$. As a check we calculate the average residence time at site 1 as given below.

$$
\left\langle T_{1}\right\rangle \simeq \int_{1}^{T_{1, \max }} \operatorname{Prob}_{L}\left(T_{1} \geq t\right) d t \sim L^{\omega_{1}} \ln \left(T_{1, \max }\right)^{1-\delta_{1}}
$$

The upper cutoff on the timescale is provided by $1 / \operatorname{Prob}_{L}\left(h=h_{\text {min }}\right)$, which is the average time interval between successive returns to the minimum height. Assuming the scaling function $g(x)$ varies as $\exp \left(-|x|^{\alpha}\right)$ for $x \ll-1$ and then putting $T_{1, \max }=\exp \left[k L^{\alpha\left(1-\omega_{1}\right)}\right]$ in the above equation, we see that $\left\langle T_{1}\right\rangle$ is proportional to $L$.

For the $1 d$ Oslo model, numerical estimate from the simulation gives $\omega_{1} \approx 0.25$. Assuming the value $\alpha \approx$ 4 (argued in section 5 ), we get $\delta_{1} \approx 0.75$. In Fig. 8 we have plotted $\operatorname{Prob}_{L}\left(T_{1} \geq t\right)$ versus a scaled variable $t / L^{\omega_{1}}$ where $\omega_{1}=0.25$ for $L=20,25,35$ and 50 and fitted the envelop formed by steps in the curves with a function $1 / x(\ln x)^{\delta_{1}}$ where $\delta_{1}=0.75$. We see that we get a reasonable fit to the data. We note that the multiplying logarithmic factor is necessary to get a good fit to $1 / t$ dependence.

\section{THE DISTRIBUTION OF RESIDENCE TIMES $T$ IN THE OSLO MODEL.}

The arguments given in the previous section are easily extended to distribution of the residence times $T_{i}$ with $i \neq 1$, and we conclude that they would also have a simi$\operatorname{lar} 1 / t$ distribution with same logarithmic correction factor which is for $T_{1}$, so long as $i$ is not near the right end.
Hence the distribution of their sum $T=\sum_{i} T_{i}$ would also be of same form.

Even though the cumulative distribution of residence times $T_{i}$ at any site $i$ has steps like structure, the stepstructure may be washed out in the sum $\sum_{i} T_{i}$.

Results of the numerical simulation for distribution of the total residence times using a total $5 \times 10^{7}$ grains are shown in Fig. 9. We see that the steps are not seen in the distribution $\operatorname{Prob}_{L}(T \geq t)$ for different values of $L$ for the range of the total residence times reached in the simulation $\left(T \leq 10^{8}\right)$. The function $\operatorname{Prob}_{L}(T \geq t)$ is much smoother than the function $\operatorname{Prob}_{L}\left(T_{1} \geq t\right)$. However for small values of $L$ (say for $L \leq 20$ ), various curves of $\operatorname{Prob}_{L}(T \geq t)$ still cross each other at large times. But for larger values of $L$, we don't see any inter-crossing of the curves in the times reached in our simulation (except at the tail where the data is less reliable due to the statistical fluctuations).

In analogy with results for the the distribution of $T_{1}$, We can expect the behaviour of the cumulative distribution $\operatorname{Prob}_{L}(T \geq t)$ to be a scaling function of $t / L^{\omega}$, where the exponent $\omega$ is different from $\omega_{1}$ defined earlier. So we write

$$
\operatorname{Prob}_{L}(T \geq t)=f\left(\frac{t}{L^{\omega}}\right)
$$

where the scaling function $f(x)$ varies as $1 /\left[x(\log x)^{\delta}\right]$ for large $x$, and the exponent $\delta$ would also be different from $\delta_{1}$ defined earlier. Using the condition that the mean residence time in the pile is equal to the mean active mass in the pile, and hence scales as $L^{2}$, can be used to determine $\delta$ in terms of $\omega$ and $\gamma$ by integrating $\operatorname{Prob}_{L}(T \geq t)$ over $t$ upto the cut-off time scale $\exp \left(\kappa L^{\gamma}\right)$. Now we get,

$$
\delta=1-(2-\omega) / \gamma
$$

In Fig. 10 we have plotted $\operatorname{Prob}_{L}(T \geq t)$ versus scaled variable $t / L^{\omega}$ where $\omega \approx 1.25[10]$. We get the value of $\delta$ approximately equal to 0.81 from Eq. (13), assuming $\gamma=3$ (argued in section 5). The fit is seen to be very good. In the numerical analysis of Christensen et. al. [5], no logarithmic factor was used, and the data was fitted with a larger effective exponent, i.e. , $1 / t^{1.22}$ decay.

\section{PROBABILITY OF MINIMUM SLOPE IN THE OSLO MODEL}

The function $\operatorname{Prob}_{L}\left(h_{1}\right)$ can be exactly calculated numerically for small $L$ using the operator algebra satisfied by addition operators 13 . We denote any stable configuration by specifying slope values at all sites from $i=1$ to $i=L$, e.g., $|122 \ldots . .21\rangle$. Whenever slope $z_{i}$ becomes 2 


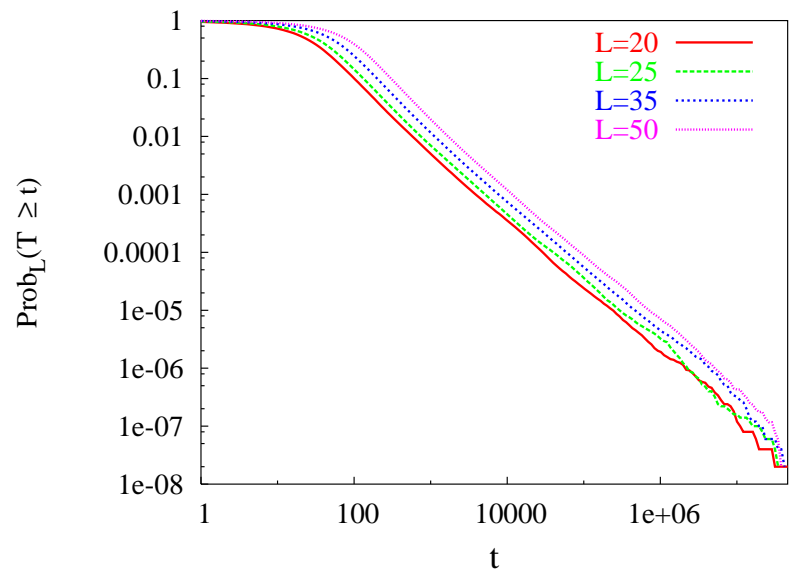

FIG. 9: The cumulative probability $\operatorname{Prob}_{L}(T \geq t)$ versus time $t$ for lattice sizes $L=8,11,15$ and 20 in the $1 d$ Oslo ricepile model. Total $5 \times 10^{7}$ grains were added.

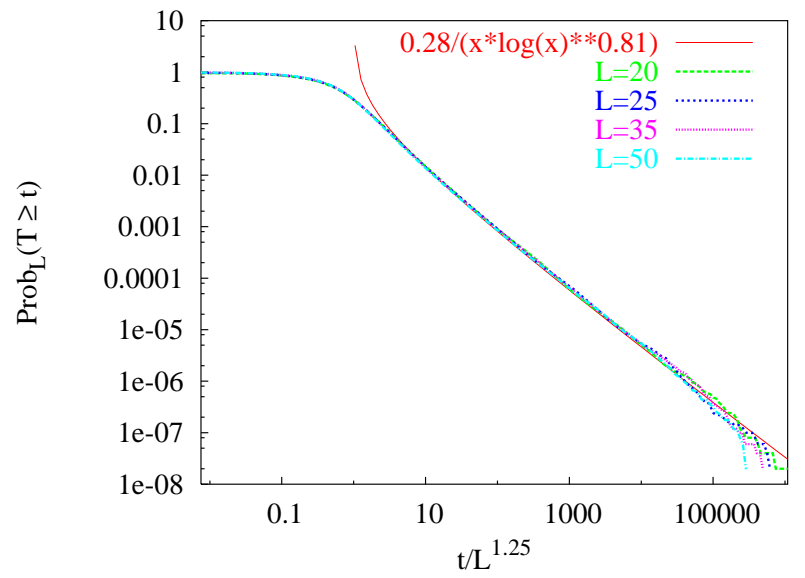

FIG. 10: Scaling collapse of $\operatorname{Prob}_{L}(T \geq t)$ versus scaled residence time $t / L^{1.25}$ in the $1 d$ Oslo ricepile model for lattice sizes $L=20,25,35$ and 50 . Total $5 \times 10^{7}$ grains were added.

after additions or toppling at site $i$, we denote such slope by $\overline{2}$, i.e., $\mid \ldots \overline{2}$... . "Bar" denotes that the site may topple or become stable with probability $q$ or $p$ respectively.

Using these two toppling rules repeatedly and the abelian property of the $1 d$ Oslo ricepile model, we can relax any unstable configurations. For example, if we relax $|\overline{2} \overline{2}\rangle$ for $L=2$, we get the following sequence, $|\overline{2} \overline{2}\rangle \rightarrow p|2 \overline{2}\rangle+q|1 \overline{2}\rangle \rightarrow p^{2}|22\rangle+p q|12\rangle+p q|1 \overline{2}\rangle+q^{2}|\overline{2} 1\rangle \rightarrow$ $\ldots \rightarrow p^{2}|22\rangle+\left(p+p^{2}\right) q|12\rangle+\left(p+p^{2}\right) q^{2}|21\rangle+(p+$ $\left.p^{2}\right) q^{3}|02\rangle+(1+p) q^{4}|11\rangle$.

The probability of maximum slope configuration (i.e., when $h_{1}=2 L$ ) can be easily calculated. We start with the unstable configuration $|\overline{2} \overline{2} \ldots \overline{2}\rangle$. The probability that no site topples in this unstable configuration is $p^{L}$ and this is the probability of the maximum slope configuration (i.e. $h_{1}=2 L$ ) in the steady state. That this

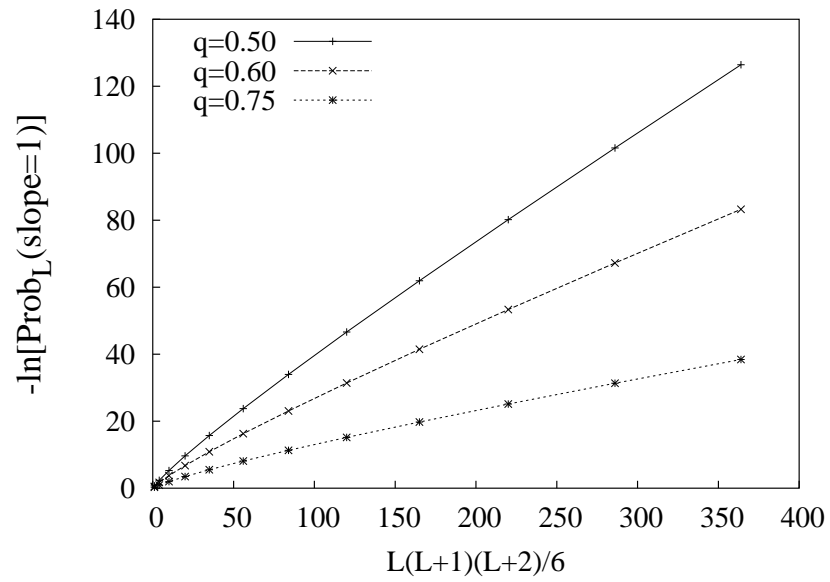

FIG. 11: Probability of occurrence of minimum slope configuration, calculated exactly, is plotted versus system sizes $L$ in the Oslo ricepile model in the semi log scale. We calculated for $L=1$ to 12 .

probability varies as exponentially with $L$ can be incorporated in the scaling hypothesis by assuming that the scaling function $g(x)$ in Eq. (1) varies as $\exp \left(-a x^{\frac{1}{1-\omega_{1}}}\right)$ for $x \gg 1$ where $a$ is a constant.

The probability of the minimum slope configuration cannot be calculated so easily. However we argue below that this probability asymptotically varies as $\exp \left(-\kappa L^{3}\right)$ where $\kappa$ is a constant.

Firstly, the above calculation for $L=2$ showed that, in the steady state, the probability of the minimum slope configuration is $\mathcal{O}\left(q^{4}\right)$. For $L=3$, we calculated this probability explicitly [19] which is $\mathcal{O}\left(q^{10}\right)$. Similar analysis, for other values of $L=1$ to 20 , shows that the probability of minimum configuration is $\mathcal{O}\left(q^{m_{L}}\right)$, where $m_{L}$ is exactly given by the formula $L(L+1)(L+2) / 6$. The coefficient of $q^{m_{L}}$ in the probability is harder to compute explicitly for large $L$. We conjecture that this simple formula holds true for all $L$. Then for sufficiently small $q$, the probability of minimum height configuration in the $1 d$ Oslo model varies as $\exp \left[-\kappa(q) L^{3}\right]$, where $\kappa(q)$ is a $q$-dependent function. Then, as there is no change in the behaviour of the Oslo ricepile expected, as a function of $q$, this behavior should persist for all non-zero $q$. For the scaling function, this would imply that $g(x)$ varies as $\exp \left[-\kappa(q)|x|^{\frac{3}{1-\omega_{1}}}\right]$ for $x \gg 1$.

We have calculated, $\operatorname{Prob}_{L}($ slope $=1)$, i.e. , the probability of the minimum slope configuration, exactly numerically for $q=0.50,0.60,0.75$ for $L=1$ to 12 and the logarithm of $\operatorname{Prob}_{L}($ slope $=1)$ has been plotted versus $\frac{L(L+1)(L+2)}{6}$ in Fig. 11 .

More specifically, consider a very low-slope unstable configuration $|11 \ldots 1 \overline{2}\rangle$ which has total $L$ number of grains with slopes 1 at all sites except at the last site with slope 2 and estimate the probability to go to the minimum slope 


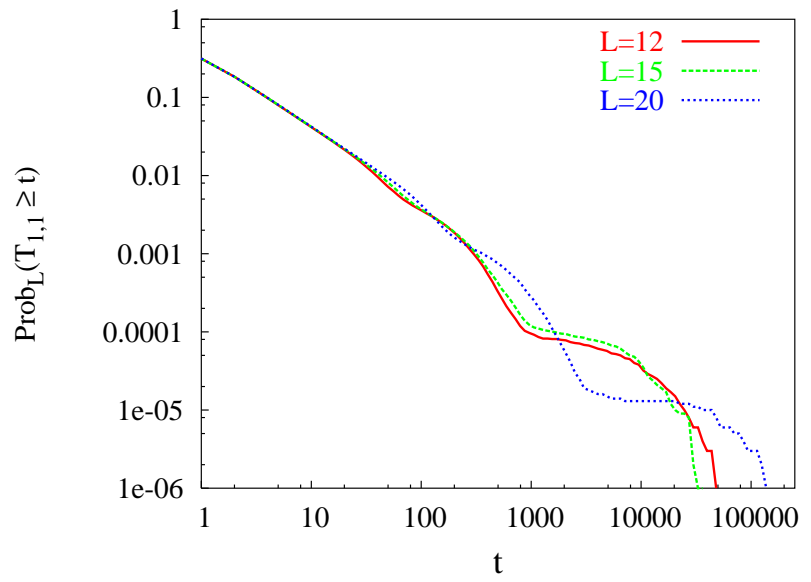

FIG. 12: The residence time distribution $\operatorname{Prob}_{L}\left(T_{1,1} \geq t\right)$ of grains at the corner site versus time $t$ in model-B for lattice sizes $L=12,15$ and 20 . Total $10^{6}$ grains were added.

from this configuration. To do this, we have to remove $L$ grains, and each grain has to be moved a distance of $\mathcal{O}(L)$ on the average. Thus we need $\mathcal{O}\left(L^{2}\right)$ steps for large $L$, and each step requires a factor $q$ in probability. Actually the probability of transition from this configuration with height $h_{m i n}+1$ to the minimum slope configuration (with height $\left.h_{\text {min }}\right)$ is $\mathcal{O}\left(q^{\frac{L(L+1)}{2}}\right)$ and the coefficient of $q^{\frac{L(L+1)}{2}}$ in this case is exactly 1 . Now the probability of minimum slope can be written in a general form as given below.

$$
\operatorname{Prob}(\text { slope }=1) \sim \exp \left[-\kappa(q) \cdot L^{3}\right]
$$

where $\kappa(0)=\infty$ and $\kappa(1)=0$. The different asymptotic behaviour of large deviations in $g(x)$ is somewhat unexpected, but has been seen in other problems, such as distribution of the large deviation of current in the asymmetric exclusion process in a ring [20].

\section{GENERALIZATION TO OTHER MODELS}

In this section we present the simulation results in other models and show that the cumulative distributions $\operatorname{Prob}_{L}\left(T_{1} \geq t\right)$ and $\operatorname{Prob}_{L}(T \geq t)$ have same 1/t power law behaviour for large $t$, but with different logarithmic corrections.

\section{Model-B : Ricepile model in two dimension.}

Now we present the simulation results for $2 d$ ricepile model. We add marked grains at the corner site, i.e., at $(1,1)$. We simulated this model choosing $p=0.75$ and $q=0.25$, and study the residence time distribution of grains at the corner site $(1,1)$. The standard deviation

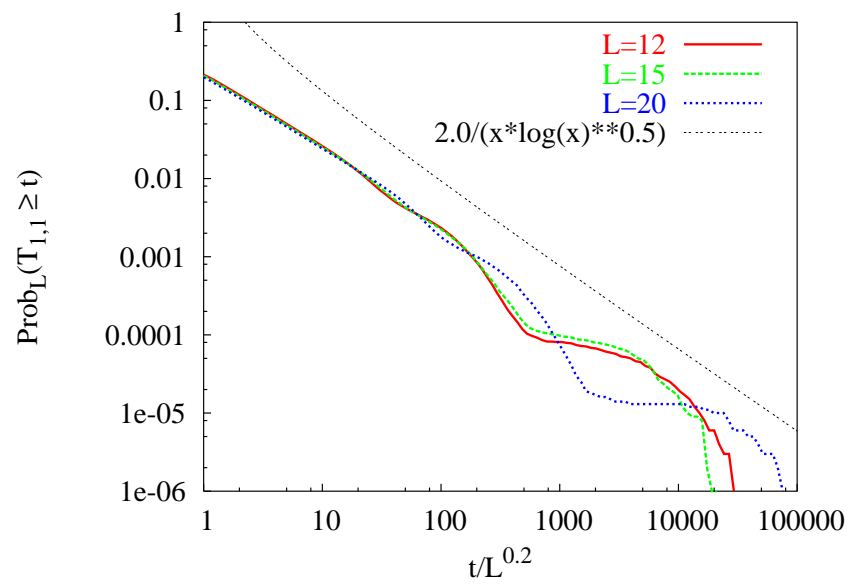

FIG. 13: The cumulative probability distribution function $\operatorname{Prob}_{L}\left(T_{1,1} \geq t\right)$ versus scaled scaled residence time $T_{1,1} / L^{0.2}$ in model-B for lattice sizes $L=12,15$ and 20 . Total $10^{6}$ grains were added.

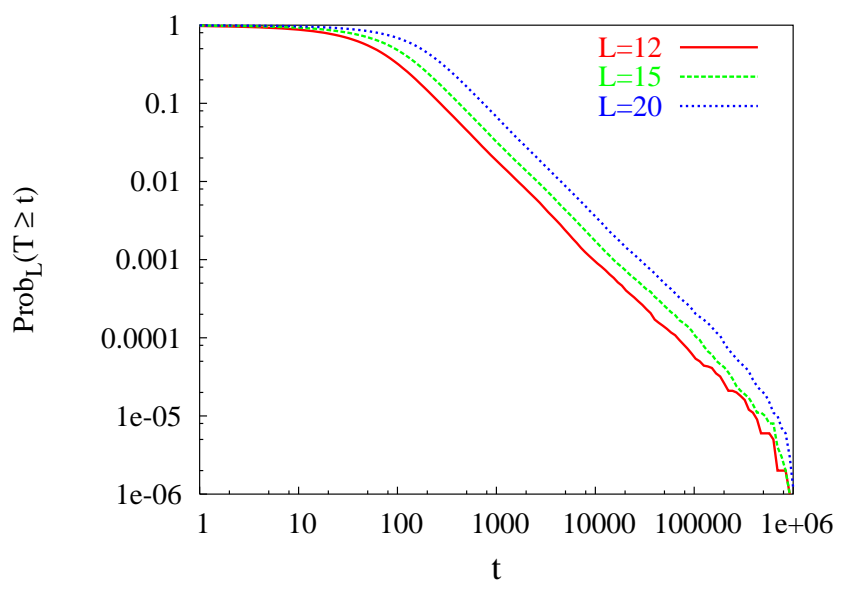

FIG. 14: The residence time distribution $\operatorname{Prob}_{L}(T \geq t)$ in model-B for lattice sizes $L=12,15$ and 20 . Total $10^{6}$ grains were added.

$\sigma_{h_{1,1}}$ of height $h_{1,1}$ at the corner site about the mean varies as $L^{\omega_{1}}$ where we estimated $\omega_{1} \approx 0.2$ from the simulation. We added total $10^{6}$ grains.

We have plotted various curves for cumulative distribution function $\operatorname{Prob}_{L}\left(T_{1,1} \geq t\right)$ of residence time $T_{1,1}$ at the corner site versus times $t$ for $L=12,15$ and 20 in Fig. 12 and we see steps like structure appearing for $t \geq 50$. Various curves for different $L$ inter-cross each other many times as seen in the $1 d$ Oslo ricepile model. In this case also, steps like structures are not log periodic as the step-length in each curve increases on log scale when going down the curve. Any simple finite size scaling does not work as in the case of the $1 d$ Oslo ricepile model. However in the Fig. 13 we plotted various cumulative distributions $\operatorname{Prob}_{L}\left(T_{1,1} \geq t\right)$ versus a scaled time 


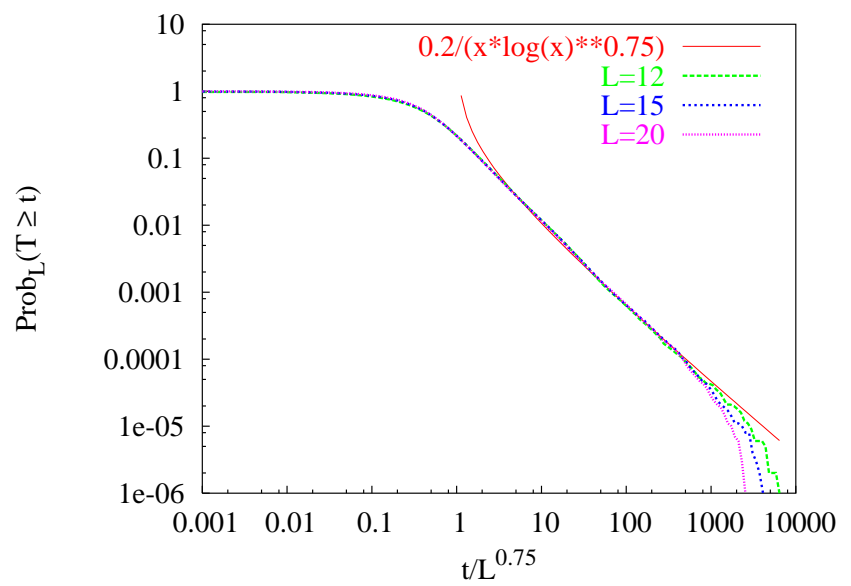

FIG. 15: Scaling collapse of various $\operatorname{Prob}_{L}(T \geq t)$ versus scaled variable $t / L^{2.0}$ in model-B for lattice sizes $L=15,20$ and 27 . Total $10^{6}$ grains were added.

$t / L^{\omega_{1}}$ with $\omega_{1}=0.2$. We see that decay of the envelop formed by various steps in different curves fit well with the function $2 /\left[x(\ln x)^{0.5}\right]$ where the logarithmic correction factor is according to Eq. (9).

Using a similar argument to the $1 d$ Oslo model, in this case, we must have $\gamma=4$. To get to the minimum slope configuration, we will have to topple $\mathcal{O}\left(L^{3}\right)$ grains and each grain $\mathcal{O}(L)$ times. As the average mass of the pile, in this case, varies as $L^{3}$, Eq. (13) is modified as given below.

$$
\delta=1-(3-\omega) / \gamma .
$$

In Fig. 14 we have plotted various distribution of the total residence time, $\operatorname{Prob}_{L}(T \geq t)$, versus time $t$ for different $L=12,15$ and 20 . In Fig. 15 we have plotted $\operatorname{Prob}_{L}(T \geq t)$ for different $L$ against the scaling variable $t / L^{\omega}$ where $\omega \approx 2.0$. Now we can estimate $\delta$ to be approximately 0.75 from the above equation. In Fig. 15 we fit the scaling function for $\operatorname{Prob}_{L}(T \geq t)$ with $0.2 /\left[x(\ln x)^{0.75}\right]$ which seems to be a reasonable fit.

\section{Model-C : The Local limited model.}

Residence time $T_{1}$ : Since in model-C, grains are added randomly everywhere in the pile, average residence time $\left\langle T_{1}\right\rangle_{h_{1}}$ of a grain added at height $h_{1}$ varies as $1 /\left[p_{1} \operatorname{Prob}_{L}\left(h_{1}\right)\right]$ according to Eq. (4). Now there will be an extra $1 / L$ factor inside the exponential in the Eq. (7). Consequently the scaling variable $\tau=\frac{t}{L^{\omega_{1}}}$ in Eq. (7) is replaced by $\tau=\frac{t}{L^{1+\omega_{1}}}$ and Eq. (17) is modified to

$$
\operatorname{Prob}_{L}\left(T_{1} \geq \tau L^{1+\omega_{1}}\right) \sim \frac{(\ln \tau)^{\frac{1-\alpha}{\alpha}}}{\tau}
$$

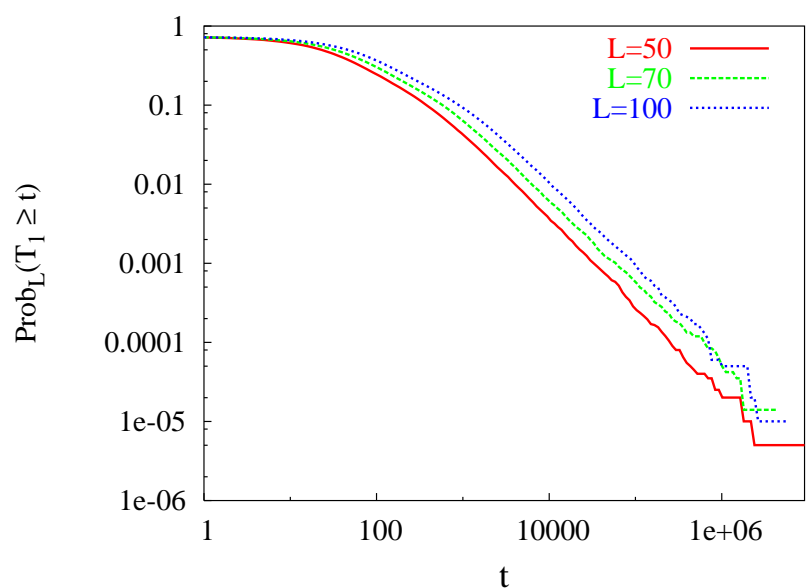

FIG. 16: The cumulative probability $\operatorname{Prob}_{L}\left(T_{1} \geq t\right)$ versus time $T_{1}$ for lattice sizes $L=25,50$ and 100 in model-C. Total $10^{6}$ grains were added.

Similarly average residence time at the first site equals to $\left\langle h_{1}\right\rangle / p_{1}$ (proof is similar as for $\left\langle T_{1}\right\rangle_{h_{1}}$ in Eq. 4) which, in this case, varies as $L^{2}$. This can be checked directly by integrating the above equation upto the cutoff time scale as done in Eq. (11).

Total number of grains added in the pile are different for different $L$ so that $10^{5}$ grains are added at the first site. The standard deviation $\sigma_{h_{1}}$ of height fluctuations at the first site varies as $L^{\omega_{1}}$ with system size $L$, where $\omega_{1} \approx 1 / 3$ 21]. We have plotted $\operatorname{Prob}_{L}\left(T_{1} \geq t\right)$ for different values of $L$ in the log-log scale in Fig. 16. We note that, unlike in the $1 d$ Oslo ricepile model, the cumulative probability here is smooth (except at the tail due to statistical fluctuations) and monotonic function of $L$ for a fixed $t$. This is due to the fact that the probability distribution $\operatorname{Prob}_{L}\left(h_{1}\right)$ of height at first site is not as sharply decaying function for $h_{1} \ll \bar{h}_{1}$ as it was in the $1 d$ Oslo model. In fact, in Fig. 17 we get a good scaling collapse of various $\operatorname{Prob}_{L}\left(T_{1} \geq t\right)$ for different $L$ using the scaled residence time $t / L^{1+\omega_{1}}$ where $\omega_{1} \approx 1 / 3$. The scaling function is fitted well with the function $1 /\left[x(\ln x)^{(\alpha-1) / \alpha}\right]$ for $x \gg 1$, taking $\alpha=2$ (see Eq. (9)).

Total residence time $T$ : In Fig. 18 we have plotted various $\operatorname{Prob}_{L}(T \geq t)$ versus residence time $t$ for lattice sizes $L=50,70$ and 100 . Total $10^{6}$ grains were added in this case. In Fig. 19 we have collapsed various $\operatorname{Prob}_{L}(T \geq t)$ for different $L$ using the scaled variable $t / L^{\omega}$ where $\omega \approx 1.5$. We fit the scaling function of the cumulative distribution with $1 / 2 x[\ln (2 x)]^{\delta}$ for $x \gg 1$ where $\delta \approx 0.63$. 


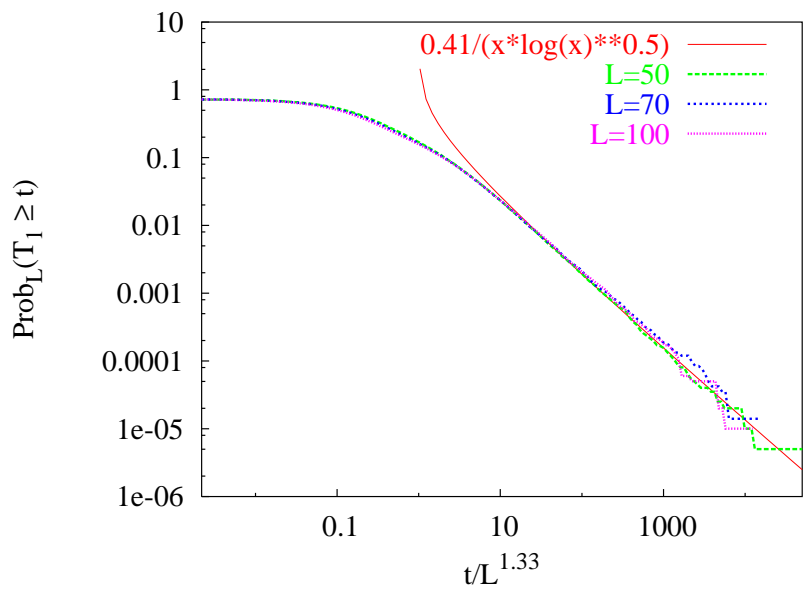

FIG. 17: Scaling collapse of $\operatorname{Prob}_{L}\left(T_{1} \geq t\right)$ for lattice sizes $L=25,50$ and 100 in model-C. Total $10^{\overline{6}}$ grains are added.

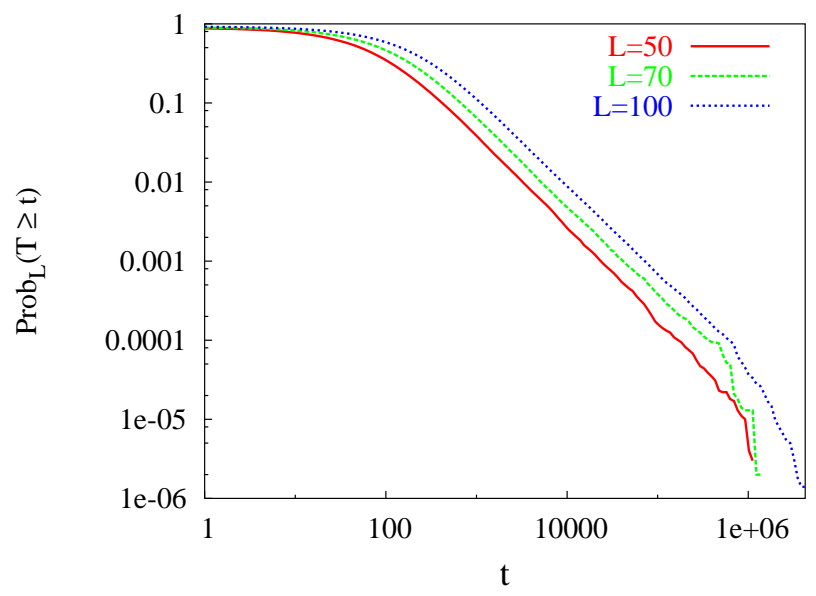

FIG. 18: The distribution function $\operatorname{Prob}_{L}(T \geq t)$ versus time $t$ in model-C for lattice sizes $L=50,70$ and 100 . Total $10^{6}$ grains were added.

\section{Model-D : Variation of the Local Limited model.}

In the model-D, the standard deviation $\sigma_{h_{1}}$ of the hight fluctuation at site 1 scales with $L$ as $L^{\omega_{1}}$ where we found $\omega_{1} \approx 0.53$. In Fig. 20 we have plotted various $\operatorname{Prob}_{L}\left(T_{1} \geq t\right)$ against the residence time $t$ at the first site for $L=75,100$ and 130 . We added $10^{4}$ grains at the first site. In Fig. 21 we have plotted $\operatorname{Prob}_{L}\left(T_{1} \geq t\right)$ versus scaled time $t / L^{1+\omega_{1}}$ with $\omega_{1} \approx 0.53$ and get a good scaling collapse of all the curves for various $L$. We fit the scaling function with $0.73 /\left[0.5 x(\ln 0.5 x)^{(\alpha-1) / \alpha}\right]$, using Eq. (9) and putting $\alpha=2$, as done in the model-C.

In Fig. 22 we have plotted various $\operatorname{Prob}_{L}(T \geq t)$ for the total residence time versus $t$ for lattice sizes $L=$ 50, 70 and 100. Total $10^{5}$ grains were added in this case. In Fig. 23 we have plotted $\operatorname{Prob}_{L}(T \geq t)$ versus scaled

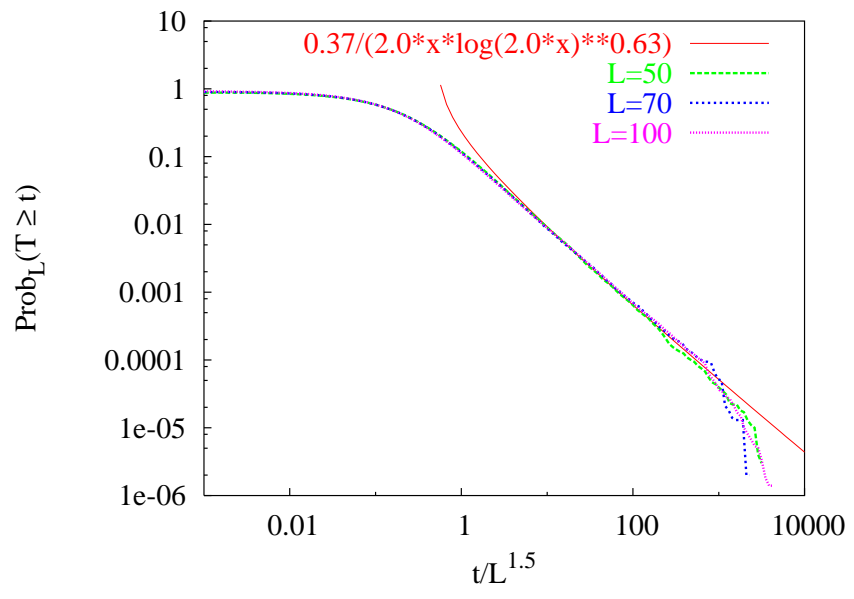

FIG. 19: Scaling collapse of $\operatorname{Prob}_{L}(T \geq t)$ versus scaled time $t / L^{1.5}$ for lattice sizes $L=50,70$ and 100 in model-C. Total $10^{6}$ grains were added.

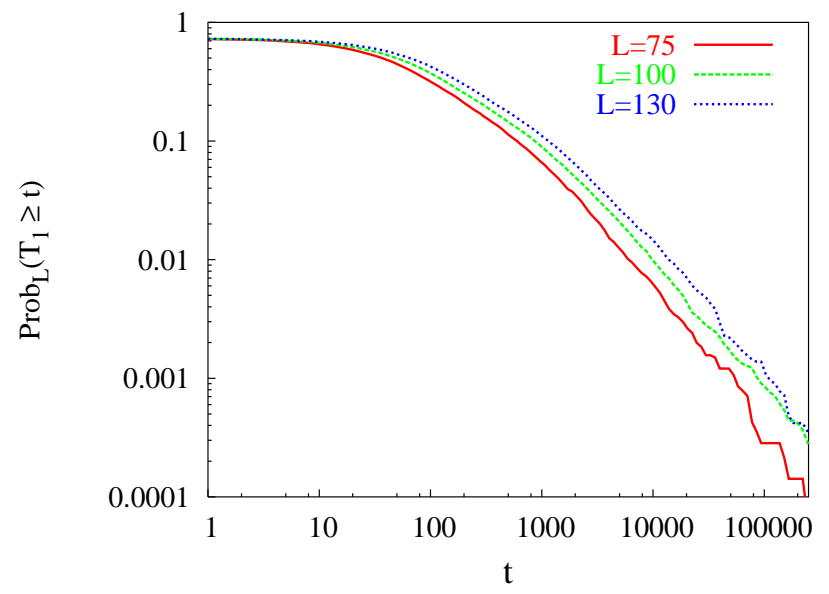

FIG. 20: The cumulative probability $\operatorname{Prob}_{L}\left(T_{1} \geq t\right)$ versus time $t$ for lattice sizes $L=75,100$ and 130 in model-D. $10^{4}$ grains were added at the first site.

time $t / L^{\omega}$ where $\omega \approx 1.5$ and we get a good collapse for the scaling function which fits reasonably well with the function $0.34 / 2 x[\ln (2 x)]^{\delta}$ for $\delta \approx 0.63$.

\section{SUMMARY AND CONCLUDING REMARKS}

To summarize, in this paper, we studied distribution of the residence times of grains in various ricepile models. We reduced the problem of finding the residence time distribution of grains at a particular site to that of determining the distribution of first return time of height at the site to the same value. The result that the probability of the residence times $T_{i}$ at site $i$ or the total residence time $T$ in the pile, being greater than or equal 


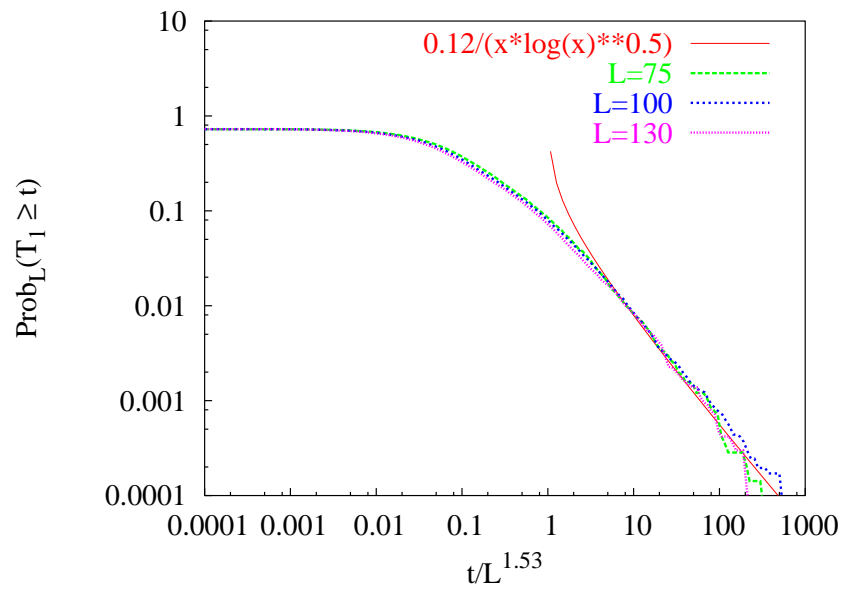

FIG. 21: Scaling collapse of $\operatorname{Prob}_{L}\left(T_{1} \geq t\right)$ for lattice sizes $L=70,100$ and 130 in model-D.

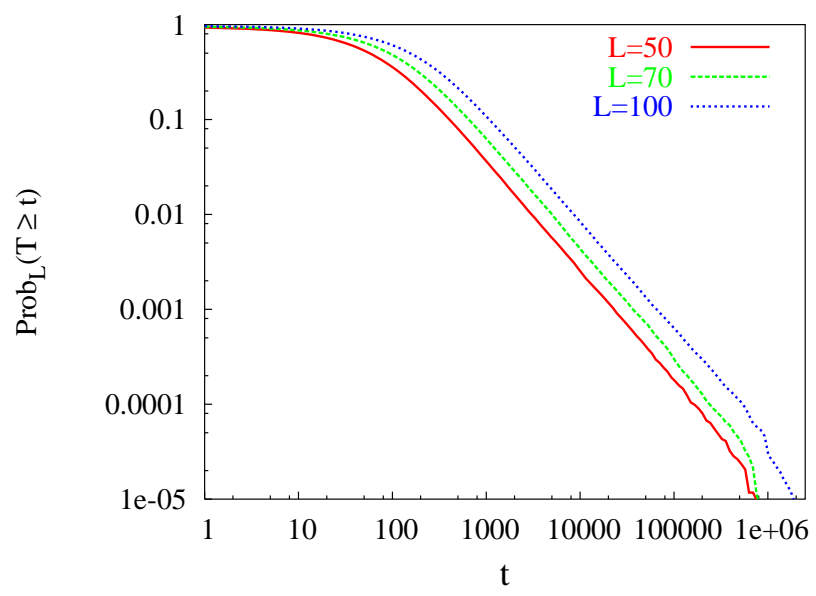

FIG. 22: The cumulative probability $\operatorname{Prob}_{L}(T \geq t)$ versus time $t$ for lattice sizes $L=50,70$ and 100 in model-D. Total $10^{5}$ grains were added.

to $t$, decay as power law $1 / t$ is valid for a large class of sandpile models, where height fluctuation at a particular site grows with the system sizes, and is independent of dimensions. It depends only on the fact that there are some deeply buried grains which come out only in rare fluctuations, i.e., slope of the pile becomes very close to the minimum slope. It is important to note that, since the total residence time $T$ is sum of $T_{i}$ 's, the probability of $T=0$ is very small, and clearly our analysis cannot predict the behaviour of the cumulative probability $\operatorname{Prob}_{L}(T \geq t)$ for $t<L^{\omega}$.

We also found that cumulative probability $\operatorname{Prob}_{L}\left(T_{1} \geq\right.$ $t)$ is non-monotonic with system size $L$ for any fixed $t$ for some of the ricepile models. The non-monotonic behaviour of the cumulative probability distribution $\operatorname{Prob}_{L}\left(T_{i} \geq t\right)$ of residence times at site $i$ with system

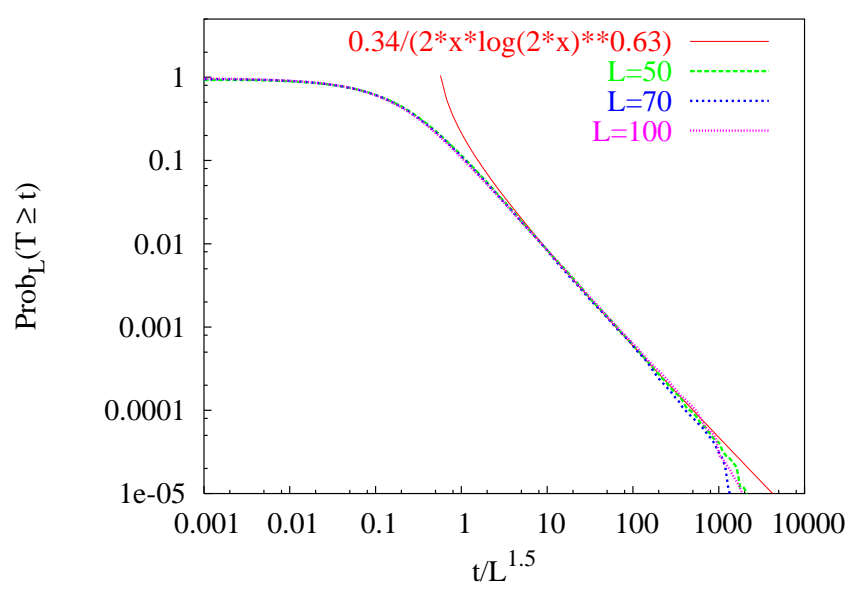

FIG. 23: Scaling collapse of $\operatorname{Prob}_{L}(T \geq t)$ against the scaled variable $t / L^{1.5}$ for lattice sizes $L=50,70$ and $100 \mathrm{in}$ model-D.

size $L$ is possible when the probability distribution function $\operatorname{Prob}_{L}\left(h_{i}\right)$, where $h_{i}$ is the height at site $i$, sharply decays for $h_{i}<\bar{h}_{i}$. However this non-monotonicity is seen only for $t \gg t^{\star}(L)$ where $t^{\star}(L)$ increases with increasing values of $L$, and hence may be harder to observe in real experiments.

It is important to note that if we change the transfer rule of grains, the distribution of residence times may change completely. The rule, chosen in this paper is called first-in-last-out rule. We may employ some other rules, such as the first-in-first-out rule, adding the grain at the top of the stack but take out grains from the bottom of the stack. A different rule would be to add and take out grain from a stack in random order. In these cases, there are no sites with deeply buried grains and the residence time distribution will be similar to that in the critical height models studied earlier by us.

[1] A. Mehta and G. C. Barker, Rep. Prog. Phys., 57, 383416 (1994). H. M. Jaeger and S. R. Nagel, Rev. Mod. Phys., 68, 1259 (1996).

[2] P. Bak, C. Tang and K. Wiesenfeld, Phys. Rev. Lett. 59, 381 (1987). P. Bak, C. Tang and K. Wiesenfeld, Phys. Rev. A 38, 364 (1988). S. R. Nagel, Rev. Mod. Phys., 64, 321 (1992).

[3] H. M. Jaeger, C. Liu and S. R. Nagel, Phys. Rev. Letts., 62, 40 (1989). G. A. Held, D. H. Solina, H. Solina, D. T. Keane, W. J. Haag, P. M. Horn and G. Grinstein, Phys. Rev. Letts., 65, 1120 (1990). J. Rosendahl, M. Vekic and J. Kelley, Phys. Rev. E, 47, 1401 (1993). P. Evesque, D. Fargeix, P Habib, M. P. Luong and P. Porion, Phys. Rev E, 47, 2326 (1993). E. Altshuler, O. Ramos, C. Martinez, L. E. Flores and C. Noda, Phys. Rev. Letts., 86, 5490 (2001).

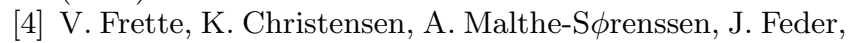


and T. J $\phi$ ssang and P. Meakin, Nature (London) 379, 49 (1996). M. Kardar, Nature (London) 379, 22 (1996).

[5] K. Christensen, A. Corral, V. Frette, J. Feder, and T. J $\phi$ ssang, Phys. Rev. Lett., 77, 107 (1996).

[6] C. M. Aegerter, R. Gunther and R. J. Wijngaarden, Phys. Rev. E, 67, 051306 (2003).

[7] H. J. Jensen, Self Organized Criticality, Cambridge University Press, Cambridge, 1998. E. V. Ivashkevich and V. B. Priezzhev, Physica A, 254, 97 (1998). D. Dhar, Physica A, 263, 4 (1999). D. L. Turcotte, Rep. Prog. Phys., 62, 1377 (1999).

[8] D. Dhar and P. Pradhan, JSTAT, online at stacks.iop.org/JSTAT/1742-5468/2004/P05002.

[9] V. Frette, Phys. Rev. Lett. 70, 2762 (1993).

[10] M. Boguna and A. Corral, Phys. Rev. Letts., 78, 4950, 1997.

[11] B. A. Carreras, V. E. Lynch, D. E. Newman and G. M. Zaslavsky, Phys. Rev. E, 60, 4770, 1999.

[12] P. Pradhan and Apoorva Nagar, cond-mat/0403769

[13] D. Dhar, Physica A, 340, 535 (2004).
[14] A. Chua and K. Christensen, cond-mat/0203260

[15] L. P. Kadanoff, S. R. Nagel, L. Wu, and S. Zhou, Phys. Rev. A, 39, 6524, 1989.

[16] A. B. Chhabra, M. J. Feigenbaum, L. P. Kadanoff, A. J. Kolan and I. Procaccia, Phys. Rev. E, 47, 3099 (1993).

[17] W. Feller, Introduction to Probability Theory, John Wiley and Sons, New York, 3rd edition, 1967, Vol. 1, Chapter $X V, X V I$.

[18] J. G. Kemeny and J. L. Snell, Finite Markov Chains, D. Van Nostrand Company, Inc., Princeton, New Jersey, 1970, Chapter $I V$.

[19] Probability of the minimum slope configurations in the steady state for $L=2$ and $L=3$ are $(1+p) q^{4}$ and $\left(1+4 p+6 p^{2}+5 p^{3}+2 p^{4}\right) q^{10}$ respectively.

[20] B. Derrida and J. L. Lebowitz, Phys. Rev. Letts., 80, 209 (1992).

[21] J. Krug, J. E. S. Socolar and G. Grinstein, Phys. Rev. A, 46, R4479 (1992). 